\section{Automatic Detection of Driving- Lane Geometry Based on Aerial Images and Existing Spatial Data}

Gl_Forum 2021, Issue 2

Page: 122 - 135

Full Paper

Corresponding Author:

jruzic@post.cz

DOI: 10.1553/giscience2021_02_s122

\author{
Jakub Rưžička and Lukáš Brưha \\ Charles University, Prague, Czechia
}

\begin{abstract}
Spatial data are a key element of geographic information systems (GIS). With the growing computational power of modern GIS, the demand for accurate and up-to-date high definition (HD) spatial data grows accordingly and increases the requirements of data acquisition. To simplify and automate the process of obtaining HD road data, several methods have been created with different approaches and stages of automation. A new method combining high resolution aerial images and existing linear road data is presented in this article. The method models roads in a vector environment at the level of single driving lanes. Object-based image analysis (OBIA) is used to identify road surface markings (RSMs) in aerial images; the geometry of RSM polygons is analysed (skeletonization, neighbourhood and context analysis, pattern recognition) in order to obtain a coherent network of driving lanes. The technique is able to distinguish automatically between solid and broken lines. The method proposed was tested and proven to satisfactorily model driving lanes, including in complex situations like junctions, roundabouts or over- or underpasses.
\end{abstract}

\title{
Keywords:
}

driving lanes, road surface markings, road geometry, aerial image analysis

\section{Introduction}

High definition (HD) spatial data are a prerequisite for sub-object-level analyses, such as identifying a location within a building, or determining the exact location of a car on a road, at the level of the specific lane on which it is travelling. Sub-object level of detail (road - object level; driving lane - sub-object level) is necessary for multiple purposes, especially within smart traffic management and autonomous driving. GPS tracks of vehicles (see e.g. Guo et al. 2007) or vehicles' on-board sensors (Gupta and Choudhary 2018) may be used to obtain HD road data. However, these mapping methods would be costly and ineffective for mapping large areas, and such mapping would not necessarily represent the car's position in a driving lane as marked on the road, but habitual lanes which drivers are accustomed to use (e.g. cutting sharp bends, or crossing lane separators in order to smooth their trajectory). A different approach is to use high resolution (aerial) imagery, where traffic lanes can be identified thanks to road surface markings (RSMs). Current segmentation and classification methods are able to identify 
the different spectral values of markings and the surrounding road surface. Geometry analysis of identified RSM polygons would follow, to obtain a consistent network of driving lanes.

Extensive research has been done in identification and extraction of roads from satellite or aerial imagery; Wang et al. (2016) may serve as an overview. Most of the research, however, has been focused on identifying roads in satellite or aerial images rather than on identifying driving lanes within roads; therefore, only a limited amount of previous research is presented here.

Bakhtiari et al. (2017), Cheng et al. (2016), Hormese and Saravanan (2016), Leninisha and Vani (2015), Máttyus et al. (2017), Shahi et al. (2015), Xia et al. (2018), Yuan et al. (2009) and Zhang et al. (2018) describe the (semi-)automatic detection of road networks in airborne imagery. Their methods vary for identifying roads, and for overcoming the problems posed by road divisions at junctions, discontinuity in shadows, or misclassified areas. Jin, Feng and Li (2009) and Jin and Feng (2010) present two different methods for detecting driving lanes in aerial imagery. However, both studies end with identifying pixels of RSMs, and no geometry analysis of the network of driving lanes follows.

The pioneering work of Baumgartner and Hinz (2003) proposed extracting driving lanes from high-resolution aerial imagery in complex urban areas. Their results, however, have limited spatial accuracy and the resulting network has a lot of gaps and overlaps. Higher spatial accuracy was achieved by Jin et al. (2012) with their method of detecting RSMs in aerial imagery using Gabor filters. They did not carry out further geometry analysis of RSMs; nor did they address fading RSMs or long gaps in RSM lines. Seo (2012), on the other hand, combined aerial images with a rasterized representation of roads, and implemented a context analysis of single pixels and their iterative grouping to form driving lanes. However, computation in a raster environment is performance-intensive, and the use of topology and context rules is limited to a close neighbourhood of pixels. More recently, Fischer et al. (2018) combined OpenStreet Map (OSM) road data with aerial imagery and detected RSMs using the Random Forest classifier and Gabor Filtering. However, their method was tested only on highways, and the authors anticipated inadequate results for other types of roads, notably in urban environments.

The main objective of the method proposed in this paper is to create a vector representation of driving lanes using aerial imagery and vector road axes, like Fischer et al. (2018). However, the method is applicable to all types of road, including junctions, roundabouts, highway crossings and over-/underpasses. Similar to the method devised by Hormese and Saravanan (2016), in our approach object-based image analysis (OBIA) is used to identify polygons of RSMs in aerial images; the RSMs detected are further analysed to create a coherent network of driving lanes (similar to Baumgartner and Hinz's (2003)). The result is a linear network of driving-lane borders (in this, it is unlike the polygons of driving lanes in Baumgartner and Hinz (2003)). The method will be capable of modelling driving lanes in places where no RSMs are visible or detected. It uses road axes as a geometry source for the driving lanes leading to more accurate gap-bridging than in Jin et al. (2012). Unlike Seo (2012), the geometry analysis is carried out in a vector environment, which leads to better computational performance and the possibility of using topology rules. The proposed method distinguishes the pattern of RSM lines (broken or solid). 


\section{Materials and methods}

The first step of the analysis is to detect RSMs in multispectral aerial images using OBIA. To extract RSM polygons, the pixel size must be smaller than or equal to the width of the narrowest RSM line, ideally at least as small as half the width. The parameters of RSM lines are defined by law or technical specification documents, and may vary from country to country. Using state-of-the-art aerial scanners, it is possible to obtain images with a spatial resolution of $2.5 \mathrm{~cm}$ (Holmes, 2012). The analysis described in this paper was carried out on multispectral (RGB) aerial imagery with a spatial resolution of $0.1 \mathrm{~m}$ (see details in Section 0).

\subsection{Image classification}

OBIA comprises two steps: image segmentation and segment classification. Various criteria can be used for segmentation, and it is possible to run the segmentation iteratively at multiple different scales. For identification of the RSMs, multi-resolution image segmentation with two iterations was used (scale factors 100 and 20), with a shape/colour ratio of $0.1 / 0.9$ and a smoothness/compactness ratio of $0.5 / 0.5$.

There are multiple parameters that can be used to classify segments (band values; geometrical, positional or textural characteristics; hierarchy; attributes, etc.); even segment-, scene- or region-related features can be used. RSMs are characterized by their colour, which leads to brightness being one of the most relevant criteria. To exclude other objects with similar brightness, geometry characteristics (width, and length:width ratio) are used, because RSMs consist of narrow lines with a specified width. As Fischer et al. (2018) suggest, roads (road axes) are used as buffers or limits to the area in which OBIA is applied, thus preventing misclassification of objects which are not located on roads. Nevertheless, redundant polygons such as cars, truck trailers, bright pavements or dust polygons (i.e. areas of dust, dirt or debris) can be misclassified and have to be removed during the geometry analysis. Polygons identified as RSMs (Figure 1) are used in the next step to detect RSM lines.

\subsection{RSM geometry detection}

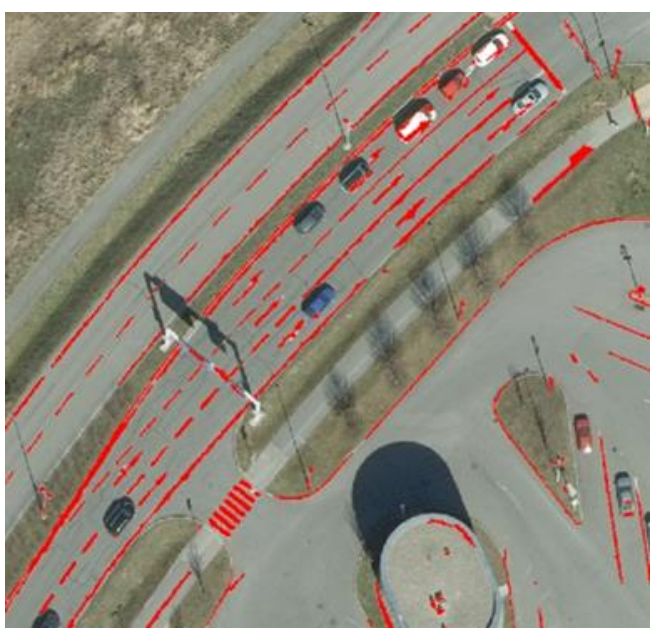

Figure 1:

Classified RSM (red). Source: Author's visualization, aerial image IPR Prague 
Classified RSMs consist of a vast number of small polygons, created during the segmentation. In order to create topologically correct RSM lines, these polygons are merged and further processed to remove geometrical inaccuracies (dangles, zigzags, irregular shapes etc.). The process of RSM geometry detection is illustrated step by step in Figure 2. Combining small neighbouring polygons into one polygon is the first step of the process. Next, agglomerates must have a main direction (i.e. a significant difference between height and length) in order to be skeletonized correctly in the following steps. Polygons which do not fulfil this condition represent a specific type of broken line and are processed separately. Agglomerates with evident main direction are thinned to the same degree (i.e. are given the same width) in order to reduce over/under-classification. Skeletonization of thinned polygons follows, and the resulting skeletons are simplified using the Douglas-Peucker algorithm (Douglas and Peucker, 1973) to prevent the resulting lines from zigzagging.

Input: polygons of classified RSM

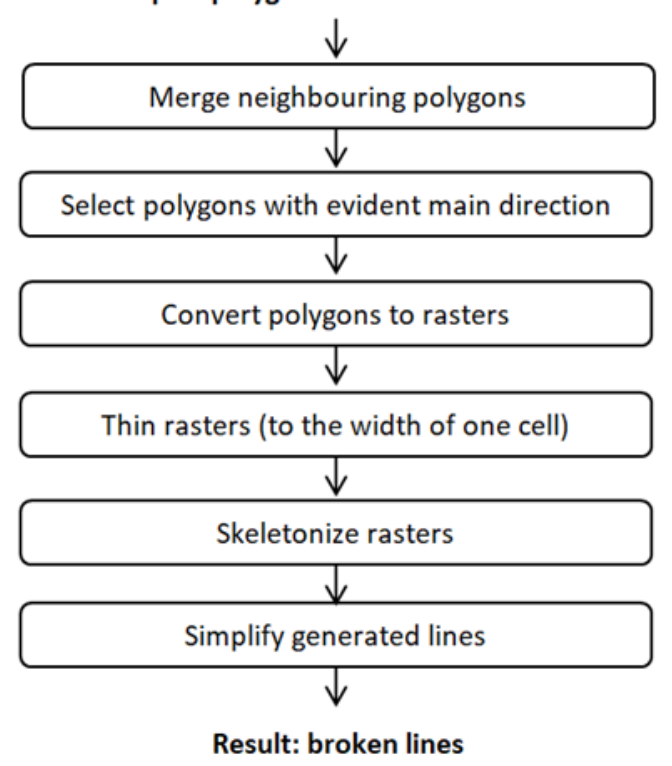

Figure 2: RSM geometry detection

\subsection{Detection of driving lanes}

RSM lines created in the previous step serve as the source of information about driving lanes, although one cannot fully rely on these as the only source. There may be cases where no RSM lines have been created (e.g. in shadows or on road patches) and additional data has to be taken into account. First, modelled RSM lines are analysed in order to create driving lanes (Fehler! Verweisquelle konnte nicht gefunden werden.); gaps (i.e. where there are no RSM lines) are then resolved.

Creating driving lanes can be simplified by interconnecting single RSM lines into continuous lines (borders of driving lanes); however, RSM line segments which are not borders of driving lanes (directional arrows, diagonal hatching, stop lines, etc.) have to be removed. This is done 
by: (1) comparing the orientation of these particular segments with the closest road axis; the dataset of roads used for the purpose must therefore have appropriate scale, completeness and correctness; (2) calculating the distance to its nearest neighbour in the main direction of the line; when a line is too isolated (i.e. has no neighbour closer than a threshold value), it is not considered to be part of a driving lane border and is removed.

Interconnecting single RSM lines (bridging gaps between broken lines or short spaces between solid line segments) is performed using buffers. Two directions of buffer (of different sizes) are used: a small buffer in line segments with a transverse direction to eliminate small differences in orientation, and a larger buffer in line segments with a longitudinal direction to bridge gaps between neighbouring segments. (The size of the longitudinal buffer is a parameter that can vary under different RSM regulations.) Buffers of all segments are merged (in case they intersect) and skeletonized. Next, the dangles of skeletons are removed and the second criterion (distance to neighbour) is applied to remove the segments which are not driving-lane borders.

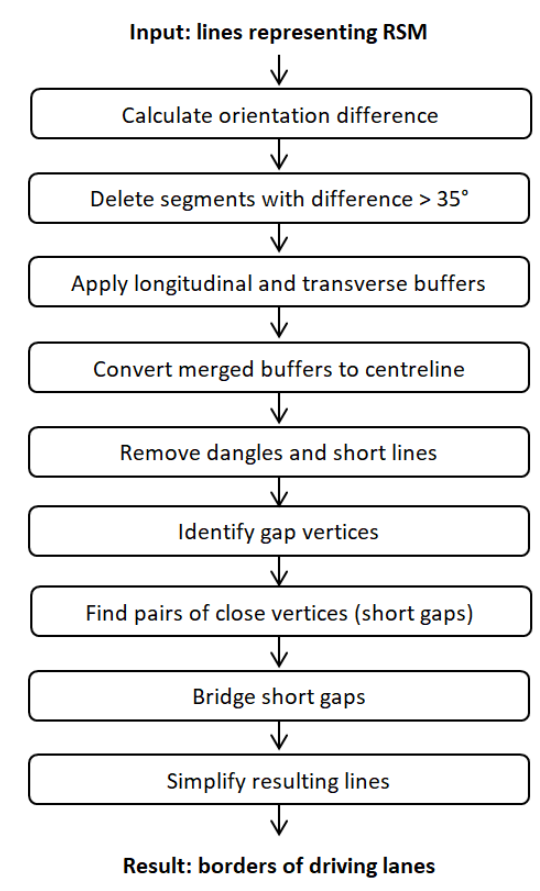

Figure 3: Driving lane detection

Two types of undesirable gap can occur in RSM borders: 'short' and 'long' ones. Gaps are identified as distances between the closest start/end vertices; the threshold distance between a short and a long gap is a numerical parameter whose value was determined experimentally as 3 metres. A short gap is created by misclassification of the aerial image (different brightness values in RSM lines, narrow shadows) or of real-life objects on the road surface (road patches, cars). Long gaps appear in places where RSM lines were not identified due to shadows (of trees or buildings), poor maintenance (fading lines), or where RSMs cannot be identified (e.g. under bridges or in tunnels). Their analysis is described in Section 0. To bridge short gaps, start/end 
vertices are interconnected (i.e. a line is created between them), and the lines forming their connections are appended to the resulting dataset. After all vertex pairs are analysed, the resulting lines are simplified in order to remove potential zigzags and smooth the resulting line.

\subsection{Bridging long gaps}

In instances of long gaps without RSMs, the border between driving lanes has to follow the direction of the road; it cannot be simplified to a straight-line connection between its vertices. In other words, if the gap is located in a bend, the line created also has to have a curved shape. Therefore, the second input dataset (vector road dataset) is necessary in this step as the geometry source.

Long gaps have to be reviewed manually, and a decision has to be made about which gaps are to be bridged (in some cases, e.g. at junctions, a gap is correct). Next, a loop over gaps to be bridged starts the bridging process (one gap per iteration is bridged). Inside the bridging loop, the start point of a gap is identified, and a perpendicular projection vector of the start point on the corresponding road axis is calculated. A point is created for each vertex of the axis; an opposite vector to the perpendicular projection vector (a 'shift' vector) is added to the points created, thus 'shifting them back' on the missing RSM line. Once the projected point gets close enough to the endpoint of the gap, the gap has been bridged, the iteration is terminated, and the loop is repeated for the next gap until all gaps are bridged (see Figure 4 for details). In the final step, all bridging lines are appended to the driving-lane borders, and all the resulting lines are simplified to remove unwanted zigzags caused by combining locations from two different datasets (identified RSM lines and road axes).

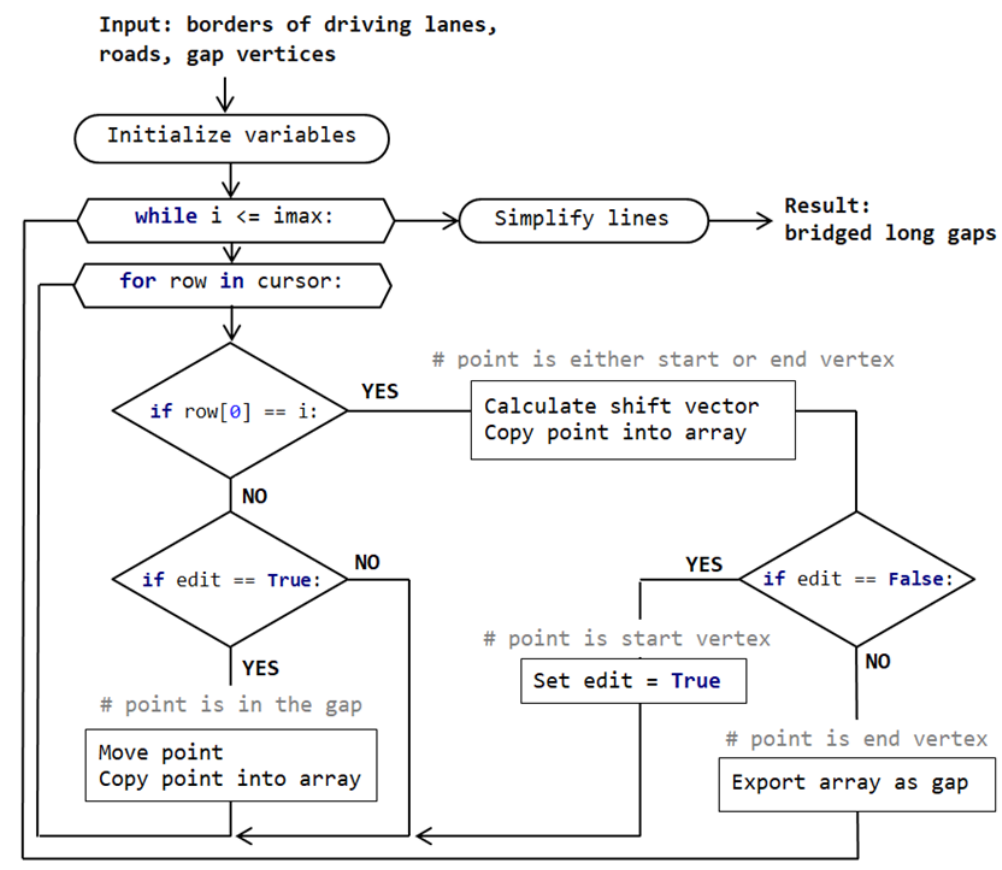

Figure 4: The loop for bridging long gaps 


\subsection{Identifying broken lines}

The last step is based on the geometry analysis performed in the previous steps and adds attribute information about the line pattern to the resulting dataset. Broken lines excluded from the original process described in Section 0 may be analysed during this step or processed as a long gap (see Section 0).

The identification of broken lines (Figure 5) involves combining the geometry of the driving lanes modelled with the pattern information of the RSM lines created. The lengths of the broken line segments are standardized, and the resulting length serves as the criterion for selecting line segments in this step. The broken lines extracted may function as a stand-alone dataset, or the pattern information can be used as an attribute in the original dataset of driving lanes.

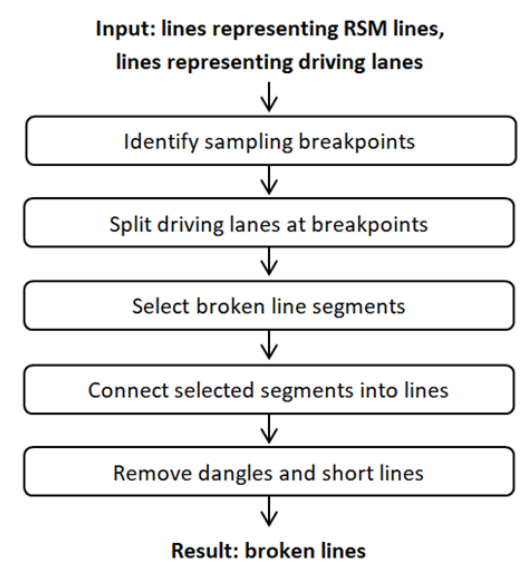

Figure 5: Identifying broken lines

\section{Testing and results}

The method was tested in a suburban area of Prague. Multiple small areas with an average size of $5,000 \mathrm{~m}^{2}$ and various types of road infrastructure (including junctions, roundabouts, bends, multiple lanes in one direction, changes in the number of lanes, overpasses and underpasses) were chosen. The main goal of the test was to find out how the method coped with different types of road infrastructure rather than to evaluate its computational costs on a large amount of data.

Aerial imagery acquired during the non-vegetative season, provided by the Institute of Planning and Development of the capital city of Prague (IPR Prague), was used to minimize the influence of tree shadows. The imagery was recorded in the visible spectrum (RGB) using the digital sensor UltraCam Eagle Mark 3 from a flight height of approximately 2,500 m, with a spatial resolution of $0.1 \mathrm{~m}$. Roads from OSM were used as the vector dataset of road axes.

Imagery was classified in e-Cognition Developer v9.5.0.; the geometry analysis was implemented in Python 3.6 using ArcPy library (ESRI 2020). The method was split into five 
algorithms which were run consecutively in order to have better control over intermediate results.

This section is divided into two parts: first, modelled RSM lines (the first step of the analysis described in Section 0) are presented and discussed; second, the driving lanes modelled (the second step of the analysis described in Section 0) are discussed.

\subsection{RSM lines}

The first step of the geometry analysis and the first output dataset are lines representing RSMs (Figure 6). The positional accuracy of the lines created was assessed using root-mean-square error (RMSE) of more than 200 randomly assigned points. A line drawn on a road surface is technically a polygon, because the lines have a width - of 0.125 or $0.25 \mathrm{~m}$, depending on type. Modelled RSM lines do not have width. The total RMSE of the modelled RSM lines is 0.126 metres.

The following shortcomings were identified, which would need to be taken into account if using this method on a large scale. However, solving these shortcomings is not within the scope of this paper.

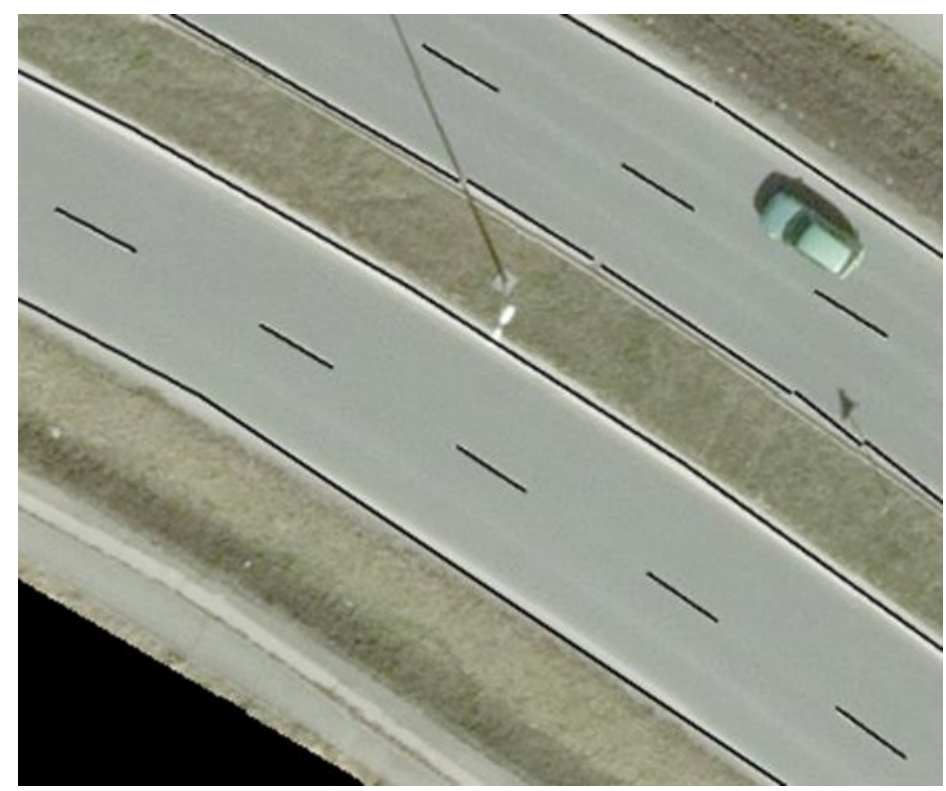

Figure 6: Modelled RSM lines (black). Source: Author's visualization, aerial image IPR Prague

\section{Diagonal hatching}

Diagonal hatching sometimes causes straight lines to oscillate (Figure 7). The oscillation was not removed during line simplification, because the lines of diagonal hatching intersect with the oscillating line. (The position of the intersection points remains unchanged.) A possible solution could be the elimination of diagonal hatching from the process at this stage. However, 
the diagonal hatching lines are part of RSMs; therefore, their elimination would not always be an appropriate solution. For such cases, lines of diagonal hatching have to be removed from the process before the simplification, then added to and interconnected with the simplified lines after the simplification.
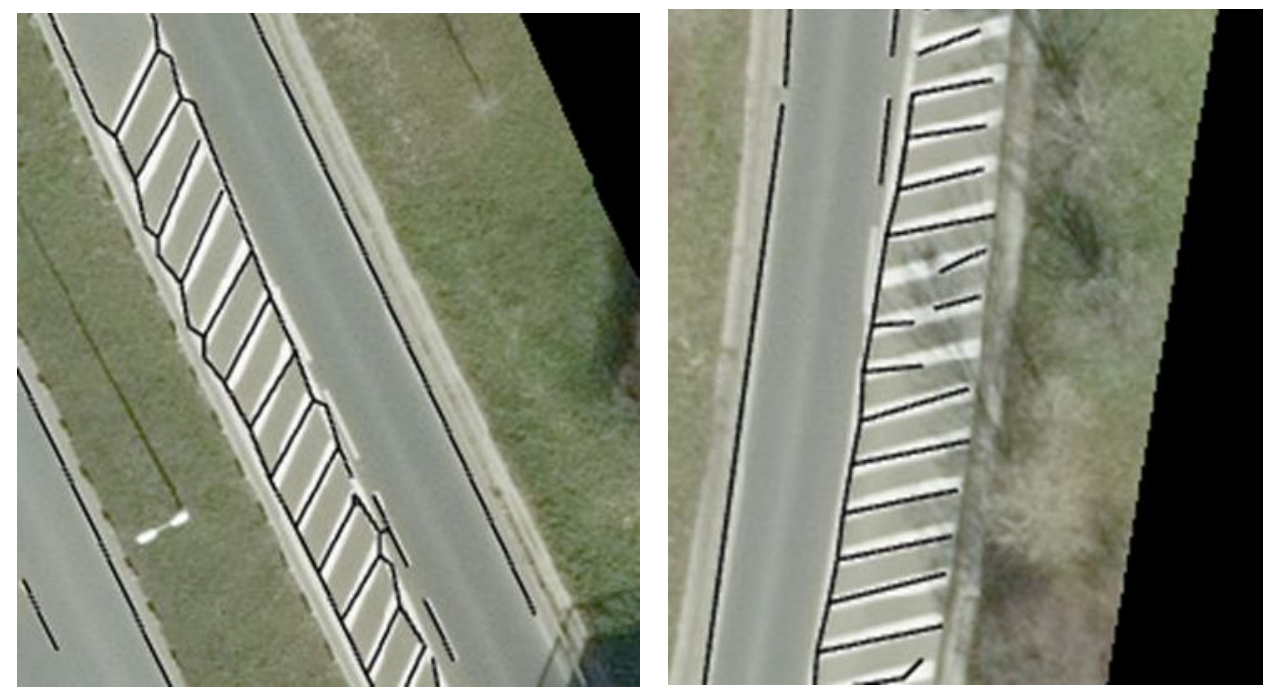

Figure 7: Oscillating line caused by diagonal hatching (on the left) and correctly created straight line (on the right). Source: Author's visualization, aerial image IPR Prague

\section{Crash barriers, railings and other misclassified objects}

Long and narrow objects with a similar brightness value are often misclassified as RSM lines during the classification, causing the RSM lines identified to be shifted closer to the edges of roads, or even away from roads. It is difficult to identify (and exclude) such objects during OBIA because their spatial and spectral characteristics are similar to those of RSMs. Consequently, such objects have to be excluded during the geometry analysis, possibly depending on the width of the driving lanes. However, this would require information about road width in the input road dataset.

\section{Shadows}

Shadows present in the original aerial image affect the brightness values of RSM lines and result in them being misclassified. Where narrow shadows are cast by streetlamps or traffic signs (see Figure 6 for examples), modelled RSM lines are broken into shorter segments or vanish completely when shaded by buildings or trees. The resulting gaps could be bridged using the same approach as for the gaps found when modelling driving lanes. However, this would require a manual review of cases to be bridged, as when bridging long gaps. 


\subsection{Driving lanes}

Driving lanes (Figure 8) were modelled with a RMSE of 0.149 metres. The driving lanes created reflect the quality of the RSMs modelled; that is, they are influenced by the shortcomings of the modelling. The procedure includes steps to bridge gaps, leading to the complete elimination of undesired gaps.
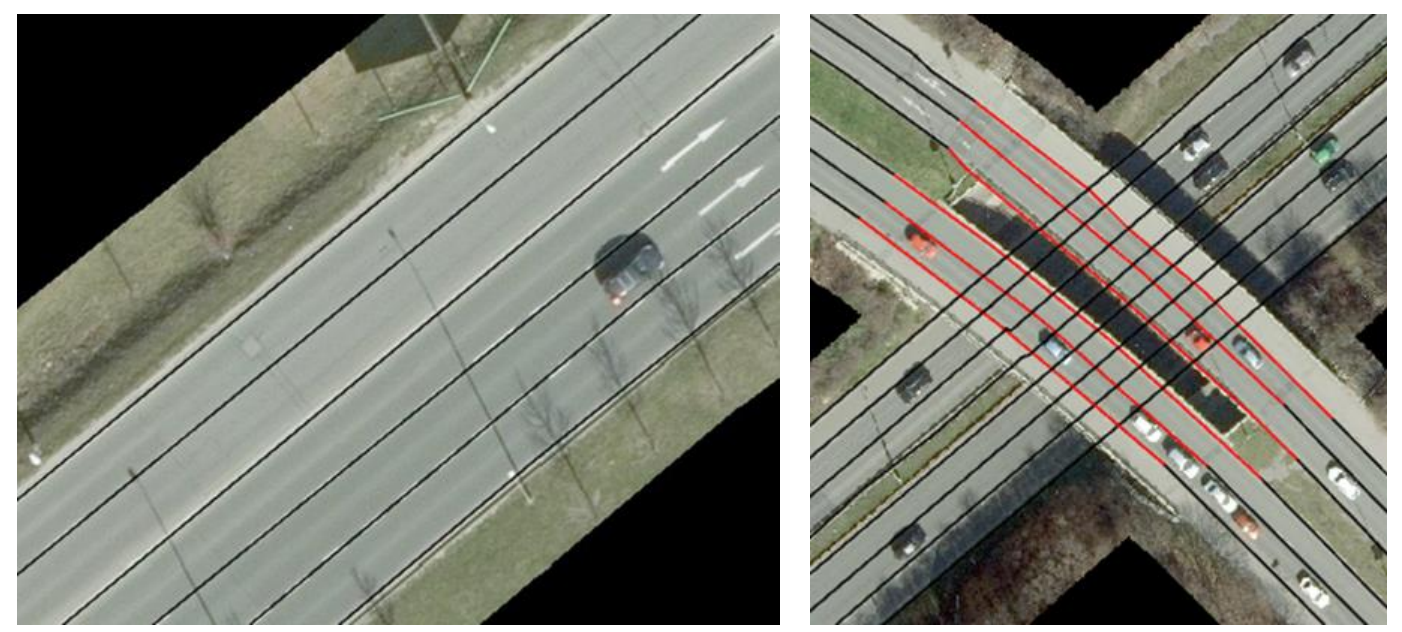

Figure 8: Modelled driving lanes, showing (on the right) an overpass with multiple levels (surface in black; bridge in red). Source: Author's visualization, aerial image IPR Prague

The problem of bridges and tunnels is solved in a semi-automated way - the location of a bridge or a tunnel has to be identified manually and is divided into multiple surface levels; each level is then analysed and modelled separately. The driving lanes created are merged into one dataset, with no differences between levels (see Figure 8, right), and with no splitting of the original dataset. It might be possible to automate the identification of the various levels, by splitting and merging processes, and through further querying of the input data in such a way as to show the locations of bridges and tunnels, either in another input layer, or as attributes of the road data being used.

Two phenomena causing inaccuracies in the resulting driving lanes were identified: undesired line oscillation, and incoherent geometry of input road lines.

\section{Undesired line oscillation}

The irregular shape of the driving lanes created is caused when an object is covering the original RSM line in the aerial image (e.g. a drainage cover and a shadow, in Figure 9 left and right respectively), resulting in a gap in the modelled RSM. In specific cases, road debris or dust polygons in the immediate vicinity of these gaps might be misclassified as RSMs and identified as the segment closest to both ends of the gaps. The misclassified objects then become incorporated into the network of drivinglanes, causing the resulting lines to oscillate. 


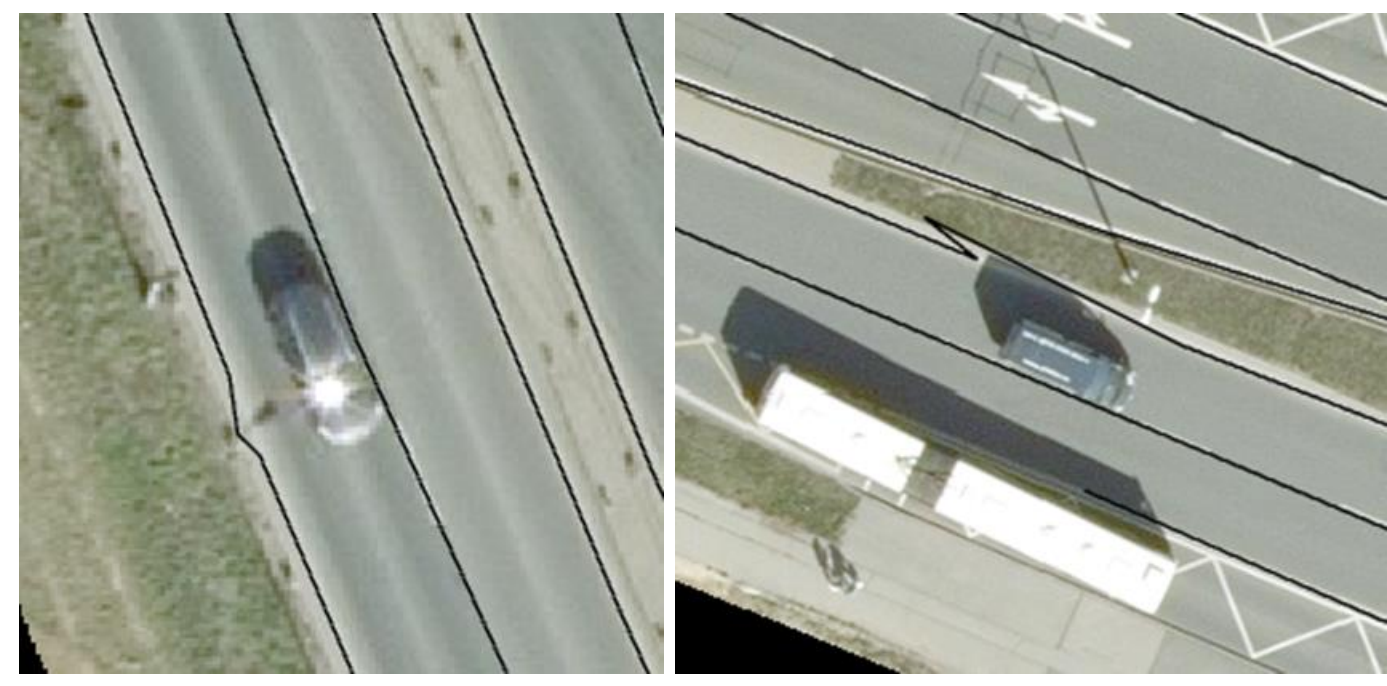

Figure 9: Line oscillation caused by a drainage cover (left) and a shadow (right). Source: Author's visualization, aerial image IPR Prague

\section{Incoherent geometry of input road lines}

Lines created in order to bridge long gaps always copy the geometry of the input road dataset. An undesired shift of the resulting driving lanes can therefore appear when road lines with an inappropriately small scale are used (Fehler! Verweisquelle konnte nicht gefunden werden.). As mentioned in Section 0, the same distance from gap to road axis (shift vector) is used; consequently, the axis must retain the same distance from the missing RSM line throughout the whole gap. An abrupt change in the number of driving lanes at a junction can lead to a change of the shift vector and thus to incorrect results (see Fehler! Verweisquelle konnte nicht gefunden werden. for visualization).
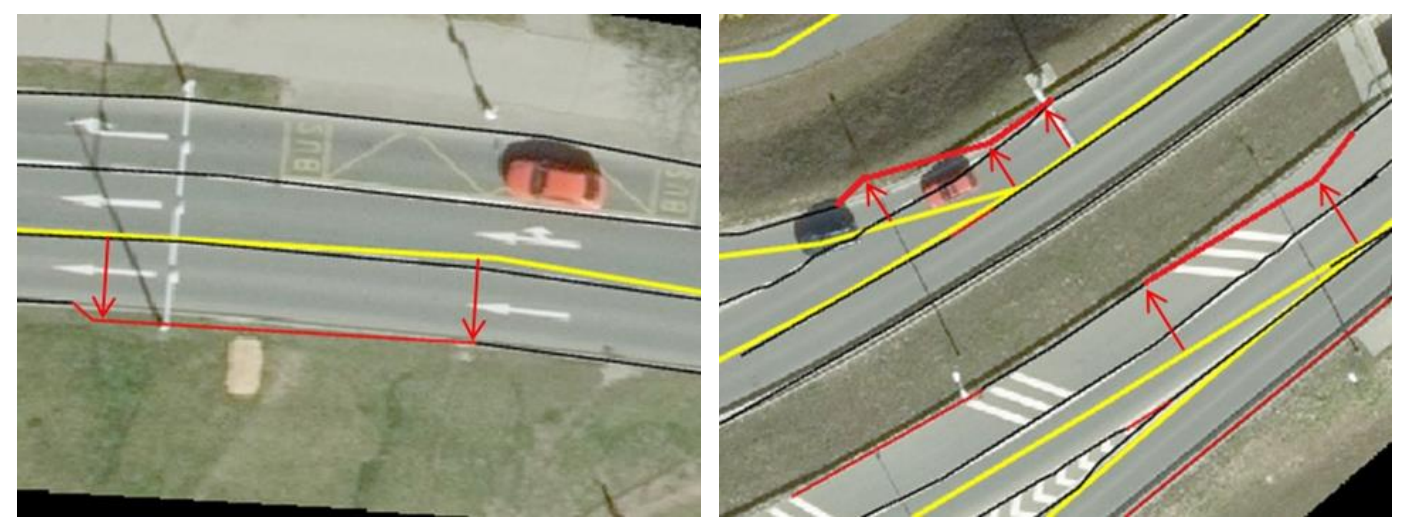

Figure 10: Bridged gaps (red) compared to input road lines (yellow): insufficient scale of input road line on the left and abrupt change of driving lanes at a junction on the right; red arrows symbolize the shift vectors. Source: Author's visualization, aerial image IPR Prague 


\section{Conclusion and outlook}

A semi-automated method of identifying driving lanes in aerial images was developed, as presented in this article. The method requires two input data sources: high-resolution aerial images in the visible spectrum (RGB) with recognizable road surface marking, and polylines of road infrastructure (axes of roads). Furthermore, both datasets should depict the road network in a coherent manner (i.e., there should be no differences between the road geometries). The method is able to handle situations in which the road is blocked from view by bridges or in tunnels, and complex situations like junctions, roundabouts or a change in the number of driving lanes.

The first step is object-based image analysis to identify RSM polygons in the input aerial image. Next, the geometry of the classified RSM polygons is analysed in order to obtain a vector (linear) representation of RSMs, from which the vector (polyline) network of driving lanes is derived. Pattern analysis of the classified RSMs identifies broken lines, and the pattern information is appended as attributes.

The proposed method has been tested in multiple case study areas covering various types of road infrastructure, including junctions, main roads with multiple driving lanes, pedestrian crossings, over- and under passes. The positional accuracy of the results modelled was evaluated using RMSE, reaching 0.126 metres for RSM lines, and 0.149 metres for driving lanes. After taking into account the width of the RSM lines, which vary between 0.125 and 0.25 metres, and the spatial resolution of the input aerial imagery ( 0.1 metres), it can be stated that the positional accuracy is sufficient.

Several shortcomings were identified during the testing. Further research is needed to improve the method in order to obtain a full working solution for the detection of driving lanes. It is possible that increasing the resolution of the input aerial imagery or the classification itself could improve the quality of the results. Another way to achieve more accurate results would be to improve the maintenance of RSMs in the first place to avoid fading lines, lines being covered by debris, or other classification errors.

This research is innovative in modelling driving lanes from aerial imagery in a vector environment. We believe that using aerial imagery to detect driving lanes is more promising than analysing GPS traces or images from on-board cameras. However, more research is required to devise a method that is suitable for use on a larger scale.

\section{Acknowledgements}

This article is based on the research for a Master's thesis carried out in 2020 by Jakub Růžička under the supervision of Lukáš Brůha at the Department of Applied Geoinformatics and Cartography of the Faculty of Science, Charles University.

Input aerial imagery was provided by the Institute of Planning and Development of the Capital City of Prague (IPR Prague), licensed under CC BY-SA 4.0

[http://creativecommons.org/licenses/by-sa/4.0/]. The road centrelines used for the research originated in OpenStreet Map (OSM), licensed under CC-BY-SA 2.0

[https://creativecommons.org/licenses/by-sa/2.0/]. 


\section{References}

Aeberhard, M., Rauch, S., Bahram, M., Tanzmeister, G., Thomas, J., Pilat, Y., Homm, F., Huber, W., Kaempchen, N. (2015): Experience, Results and Lessons Learned from Automated Driving on Germany's Highways. IEEE Intelligent transportation systems magazine, 7(1), 42-57. DOI: 10.1109/MITS.2014.2360306

Bakhtiari, H. R. R., Abdollahi, A., Rezaeian, H. (2017): Semi-automatic road extraction from digital images. The Egyptian Journal of Remote Sensing and Space Sciences, 20, 117-123. DOI: 10.1016/j.ejrs.2017.03.001

Baumgartner, A., Hinz, S. (2003): Automatic extraction of urban road networks from multi-view aerial imagery. ISPRS Journal of Photogrammetry \& Remote Sensing, 58, 83-98. DOI: 10.1016/S09242716(03)00019-4

Cheng, G., Zhu, F., Xiang, S., Wang, Y., Pan, Ch. (2016): Accurate urban road centerline extraction from VHR imagery via multiscale segmentation and tensor voting. Neurocomputing, 205, $407-$ 420. DOI: $10.1016 /$ j.neucom.2016.04.026

Douglas, D. H., Peucker, T. K. (1973): Algorithms for the Reduction of the Number of Points Required to Represent a Digitized Line or Its Caricature. The Canadian Cartographer, 10(2), 112122

ESRI, Environmental Systems Research Institute (2020): ArcGIS Pro Python reference. Redlands, California. Retrieved from https://pro.arcgis.com/en/pro-app/latest/arcpy/main/arcgis-proarcpy-reference.htm

Fischer, P., Azimi, S. M., Roschlaub, R., Krauß, T. (2018): Towards HD Maps from Aerial Imagery: Robust Lane Marking Segmentation Using Country-Scale Imagery. ISPRS International Journal of Geo-Information, 7 (458), 1-14. DOI: 10.3390/ijgi7120458

Guo, T., Iwamura, K., Koga, M. (2007): Towards high accuracy road maps generation from massive GPS Traces data. 2007 IEEE International Geoscience and Remote Sensing Symposium Conference Proceedings, 1, 667-670. DOI: 10.1109/IGARSS.2007.4422884

Gupta, A., Choudhary, A. (2018): A Framework for Camera-Based Real-Time Lane and Road Surface Marking Detection and Recognition. IEEE Transactions on Intelligent Vehicles, 3(4), 476 485.DOI: $10.1109 /$ TIV.2018.2873902

Holmes, O. (2012): High Resolution Digital Aerial Imagery vs High Resolution Satellite Imagery Part 1. Retrieved from https://aerometrex.com.au/technical/high-resolution-digital-aerialimagery-vs-high-resolution-satellite-imagery-part-1/

Hormese, J., Saravanan, C. (2016): Automated Road Extraction From High Resolution Satellite Images. Procedia Technology, 24, 1460-1467. DOI: 10.1016/j.protcy.2016.05.180

Jin, H., Feng, Y. (2010): Automated road pavement marking detection from high resolution aerial images based on multi-resolution image analysis and anisotropic Gaussian filtering. Proceedings of the 2nd International Conference on Signal Processing Systems (ICSPS), 1, 337-341. DOI: 10.1109/ICSPS.2010.5555636

Jin, H., Feng, Y., Li, Z. (2009): Extraction of road lanes from high-resolution stereo aerial imagery based on maximum likelihood segmentation and texture enhancement. Digital Image Computing: Techniques and Applications. Conference proceedings. 1, 271-276. DOI: 10.1109/DICTA.2009.52

Jin, H., Miska, M., Chung, E., Li, M., Feng, Y. (2012): Road Feature Extraction from High Resolution Aerial Images Upon Rural Regions Based on Multi-Resolution Image Analysis and Gabor Filters. In Escalante, B.: Remote Sensing - Advanced Techniques and Platforms. China: InTech, Shanghai.

Leninisha, S., Vani, K. (2015): Water flow based geometric active deformable model for road network. ISPRS Journal of Photogrammetry and Remote Sensing, 102, 140-147. DOI:

10.1016/j.isprsjprs.2015.01.013 
Máttyus, G., Luo, W., Urtasun, R. (2017): DeepRoadMapper: Extracting Road Topology from Aerial Images. 2017 IEEE International Conference on Computer Vision (ICCV) Proceedings, 34583466. DOI: 10.1109/ICCV.2017.372

Seo, W.-Y. (2012): Augmenting Cartographic Resources and Assessing Roadway State for Vehicle Navigation. PhD Thesis, April 2012, The Robotics Institute, Carnegie Mellon University, Pittsburgh, PA 15213, USA.

Shahi, K., Shafri, H. Z. M., Taherzadeh, E., Mansor, S., Muniandy, R. (2015): A novel spectral index to automatically extract road networks from WorldView-2 satellite imagery. The Egyptian Journal of Remote Sensing and Space Sciences, 18, 27-33. DOI: 10.1016/j.ejrs.2014.12.003

Wang, W., Yang, N., Zhang, Y., Wang, F. (2016): A review of road extraction from remote sensing images. Journal of Traffic and Transportation Engineering (English Edition), 3 (3), 271-282. DOI: 10.1016/j.jtte.2016.05.005

Xia, W., Zhang, Y., Liu, J., Luo, L., Yang, K. (2018): Road Extraction from High Resolution Image with Deep Convolution Network - A Case Study of GF-2 Image. 2nd International Electronic Conference on Remote Sensing, Proceedings 2 (325). DOI: 10.3390/ecrs-2-05138

Yuan, J., Wang, D., Wu, B., Yan, L., Li, R. (2009): Automatic Road Extraction from Satellite Imagery Using LEGION Networks. Proceedings of International Joint Conference on Neural Networks, 1, 3471-3476. DOI: 10.1109/IJCNN.2009.5178605

Zhang, Z., Zhang, X Sun, Y., Zhang, P. (2018): Road Centreline Extraction from Very-HighResolution Aerial Image and LiDAR Data Based on Road Connectivity. Remote Sensing, 10 